\title{
CARACTERÍSTICAS HISTOPATOLÓGICAS DE UM TUMOR ESTROMAL GASTROINTESTINAL
}

\author{
* Juliana Machado Amorim ' \\ Francisco de Assis Cavalcanti Neto" \\ Ruanna Lamille Estrela e Silva II \\ Raimundo Sales Filho III
}

\section{RESUMO}

O tumor estromal gastrintestinal (GIST) é um tipo de tumor que pode se desenvolver ao longo de todo o tubo digestivo. Possui causa desconhecida e sua ocorrência se dá nas diversas faixas etárias, sendo mais comum nos indivíduos de maior idade. O portador da enfermidade é assintomático no início do quadro, desenvolvendo sintomas apenas em estágios mais avançados. Devido ser um tumor bastante incomum, considerou-se o estudo dessa patologia a fim de ampliar o conhecimento sobre esta morbidade, que muitas vezes chega a ser desconhecida pelos profissionais da saúde. O objetivo do estudo é relatar achados histopatológicos de uma paciente diagnosticada com Tumor Gastrointestinal de origem Estromal. Foram analisados o prontuário médico do paciente e lâmina histológica. O relato de caso foi realizado em uma Clínica de Patologia particular da cidade de João Pessoa - PB. Observou-se presença de células de núcleo alongado, tumor, em parte da lâmina, restrito a camada muscular da mucosa e, em parte, invadindo a camada suprajacente, além de contar, com área de necrose. O resultado pôde ser confirmado por meio do exame da imuno-histoquímica, que mostrou presença dos marcadores CD117 e CD34. A associação da história clínica dos pacientes, exames laboratoriais e de imagem, junto ao anatomopatológico ainda não fecham de forma definitiva o diagnóstico, necessitando de imunohistoquímica com pesquisa de CD-117(c-kit). O GIST é um tumor silencioso, cuja agressividade é variável e que necessita de diagnóstico precoce, enquanto seu estudo for limitado, a sua detecção continuará muitas vezes sendo tardia e acabará por prejudicar os desfechos dos pacientes.

PALAVRAS-CHAVE: Saúde da Mulher. Menopausa. Profissionais da Saúde.

Fonoaudióloga, Mestre em saúde da Família. Docente, Faculdade de Medicina Nova Esperança, FAMENE. I Departamento de Medicina. CEP: 58067-698, João Pessoa,Paraíba, Brasil.

*Autor correspondente: juliana.amorim@famene.com.br ORCID ID: 0000-0002-7921-6618

Discente do curso de Medicina da Faculdade de Medicina Nova Esperança, FAMENE. Departamento de Medicina. CEP: 58067-698, João Pessoa,Paraíba, Brasil. Orcid: 0000-0002-0419-7109; 0000-0001-7668-382X Médico, Doutor em Saúde Pública. Professor Adjunto I, Universidade Federal da Paraíba. CEP: 58013-420, João Pessoa,Paraíba, Brasil. Orcid: 0000-0002-1071-4854 


\section{INTRODUÇÃO}

Os tumores do estroma gastrointestinal (GIST), são as neoplasias mais recorrentes do trato digestivo. Originados das células de Cajal $^{1}$, pertencem ao plexo mioentérico, localizados entre as camadas musculares longitudinal e circular da mucosa na parede do tubo gastrointestinal. Esse tipo de tumor encontra-se principalmente, na camada submucosa ou muscular própria e são revestidos por uma pseudocápsula. ${ }^{2}$

No geral, são tumores de baixa malignidade, mas com alto potencial de recidiva local e com capacidade metastáticas, por isso o tratamento deve ser radical e seguir critérios de ressecção oncológicos. 3

Os pacientes com GIST evoluem, em cerca de 30\% das vezes, de forma assintomática. As manifestações clínicas mais comuns são sangramento apresentado na porção mais alta do trato gastrointestinal (GI), incluindo hematêmese, melena ou sangue oculto nas fezes, em decorrência de úlceras na mucosa. As outras manifestações clínicas incluem dor abdominal ou massa palpável. O GIST apresenta-se de forma maligna em 25 a $30 \%$ dos casos por ocasião do seu diagnóstico inicial, pelo critério de metástases ou invasão de órgãos ou estruturas adjacentes. Por ser uma enfermidade muitas vezes silenciosa, seu diagnóstico precoce é importante a fim de evitar sua evolução para formas mais agressivas. Em casos de suspeita o exame histopatológico se faz extremamente necessário para o diagnóstico da lesão. ${ }^{2}$

Os sintomas clínicos são inespecíficos e dependem basicamente da localização e tamanho da lesão. Dor abdominal, plenitude gástrica obstrução intestinal, sendo muitas vezes achados incidentais em exames de tomografia, ressonância magnética e endoscopia. 4 Podem atingir grandes dimensões, com tamanho variando, usualmente, entre 3 e $10 \mathrm{~cm}$, por terem crescimento predominantemente extraluminal, raramente causam sintomas obstrutivos. 5

Um tumor mesenquimal, como o GIST, pode ter origem em tecidos de apoio: muscular, cartilaginoso, adiposo, ósseo, etc. Acredita-se que a origem histológica dos GIST seja as células intersticiais de Cajal e os locais mais comuns são as paredes circundantes do lúmen do trato gastrointestinal. O diagnóstico definitivo é dado por meio do exame histopatológico e pela imuno-histoquímica. Estes tumores são caracterizados pela expressão de um receptor de membrana mutante com atividade de tirosina quinase (kit c), que é evidenciado pela imuno-histoquímica (IHC) com o anticorpo CD-117, que define uma proliferação celular não regulada. 5

Em relação ao aspecto anatomopatológico, localizam-se principalmente na submucosa ou muscular própria, são revestidos por uma pseudocápsula e são bem vascularizados. Os principais tipos histológicos são: fusocelular em $75 \%$, epitelioides em $8 \%$ e mistos em $15 \%$ dos casos. A gradação histopatológica é dada de acordo com a atividade mitótica. São classificados como baixo grau, os tumores com menos de 5 mitoses/50 campos de grande aumento (CGA) e, alto grau, os acima deste valor. ${ }^{2}$

A cirurgia continua a ser a única modalidade que pode oferecer uma cura permanente do GIST. A ressecção cirúrgica completa é o tratamento inicial para GISTs primários, quando o risco de morbidade e morte por cirurgia é aceitável, evitando rupturas tumorais e lesões na pseudocápsula. Os objetivos da cirurgia incluem ressecção completa 
com margens negativas macroscópicas e microscópicas e preservação funcional por ressecção em cunha, quando aplicável. 5

Tendo em vista a característica silenciosa da doença, o diagnóstico precoce deve ser um alvo do GIST, portanto este artigo tem como objetivo relatar

\section{MATERIAL E MÉTODOS}

\begin{abstract}
O presente relato traz um estudo clínico observacional com abordagem qualitativa, utilizado para descrever o diagnóstico do tumor gastrointestinal de origem estromal. Foi realizado no Instituto de Patologia e Citologia Dr. Ely Chaves, da cidade de João Pessoa - PB.

Para estudo, foi feita a análise do
\end{abstract}

\section{RELATO DE CASO}

Foi avaliado exame anatomopatológico de um tumor do tipo GIST (no estroma gastrointestinal) de um paciente do sexo masculino, cujas informações pessoais do foram omitidas, sendo relatado apenas os dados, referentes às características da lesão propriamente dita. O material estudado tratava-se de um segmento gástrico de 6,5 $\mathrm{cm}$ de serosa lisa e transparente. A mucosa apresentava uma lesão abaulada exibindo ulceração central de aproximadamente 4,0 $\mathrm{cm}$ de diâmetro, que correspondia a massa intramural formada por tecido acastanhado e homogêneo. Os limites profundos do tumor mostravam-se rentes a superfície serosa.

No exame imuno-histoquímico foi utilizado o método da estreptavidina-biotinaperoxidase $(A B C)$, desenvolvido por Guesdon, achados histopatológicos de uma paciente diagnosticada com Tumor Gastrointestinal de origem Estromal, a fim de apresentar a importância desta entidade, para que possa ser diagnosticada em momentos mais precoces de sua evolução natural.

prontuário de um paciente com diagnóstico de tumor gastrointestinal estromal. Assim, foi possível colher as informações das lesões macroscópicas e microscópicas. Foram analisadas informações do ensaio imunohistoquímico do paciente. As análises feitas no prontuário e exame do paciente foram comparadas com a literatura atual.

Ternynck e Avrameas (1979), anticorpos monoclonais e policlonais, tendo feito aferição por controles positivos e negativos. Encontraram-se marcadores ki-67 positivo, CD117 e CD34, confirmando o diagnóstico de tumor de estroma gastrointestinal. A actina anti-músculo liso e proteína $\mathrm{S} 100$ testaram negativos. O índice proliferativo (ki-67) mostrou o equivalente a duas mitoses.

O tumor estudado apresentavase não encapsulado e localizado abaixo da camada muscular da mucosa (Figura 1-A) denunciando, em algumas regiões, sinal de infiltração para a camada mucosa, como pode ser visto na Figura 1-B, sendo observado à esquerda da imagem um segmento da camada muscular e à direitaa presença do tumor infiltrando a camada superior. 


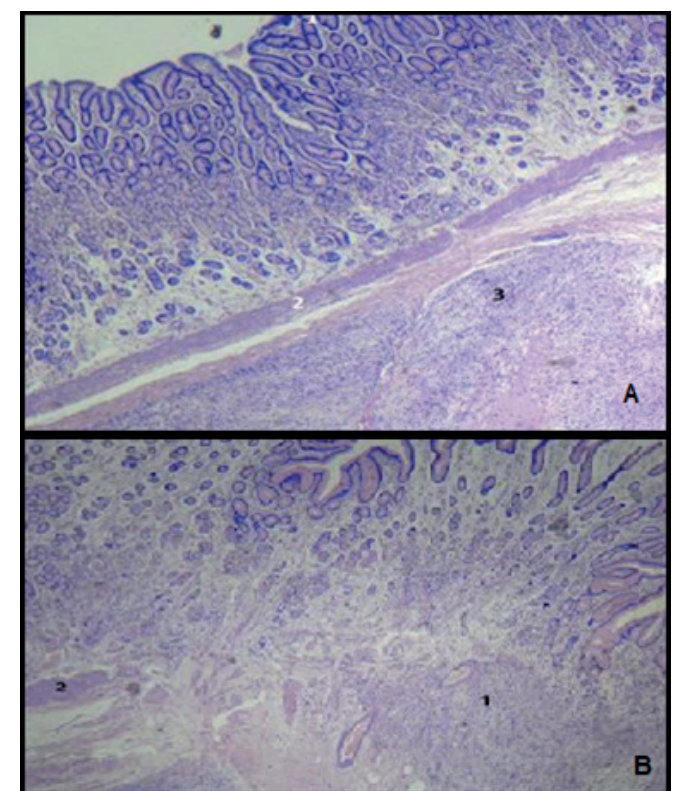

FIGURA 1: Exame histopatológico de estômago com presença de GIST. A) 1-Camada mucosa; 2-Camada muscular da mucosa; 3 - Tumor (GIST). B) 1-Tumor (GIST) infiltrando a camada muscular; 2-Camada muscular da mucosa após ser transpassada pelo GIST.

Na Figura 2-A, é possível observar que as células do tumor, apresentam núcleo alongado e, por fim, na Figura 2-B, é possível observar, além da invasão da mucosa, uma região de necrose no canto superior direito da imagem. As margens cirúrgicas profundas coincidem com as bordas da lesão. No estudo histológico não foram observadas mitoses.

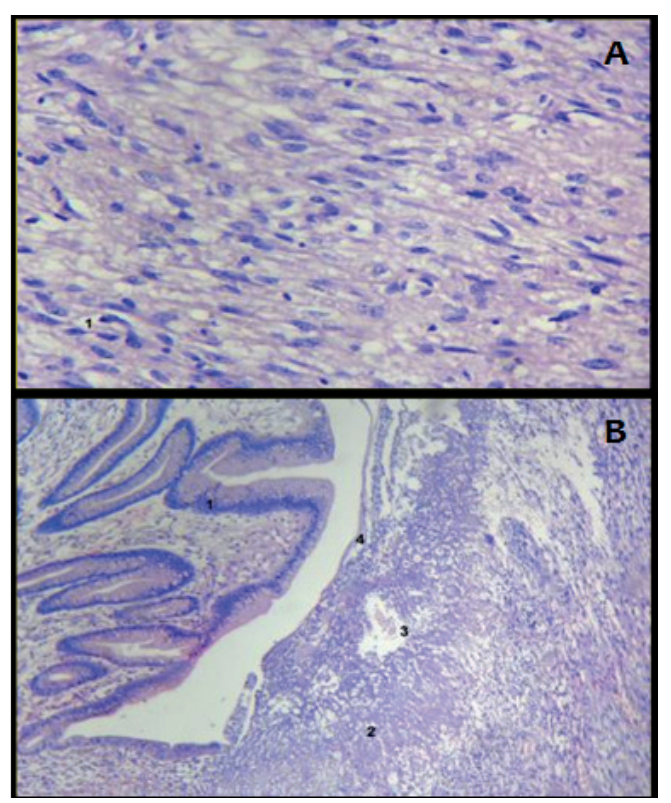

FIGURA 2: Corte histológico do estômago. A) 1 - Tumor (GIST) infiltrando a camada muscular, com presença de núcleos fusiformes; B) 1-Camada Mucosa; 2-Massa tumoral invadindo camada mucosa; 3-Área de necrosada; 4-Ulceração. 


\section{DISCUSSÃO}

Os tumores estromais gastrintestinais são as neoplasias mesenquimais que foram descritas pela primeira vez em 1998, sendo anteriormente, denominadas de neoplasias de músculo liso, leiomiomas, leiomiossarcomas e leiomioblastomas. No exame histopatológico do referido caso foram detectadas células de aspecto fusiformes e epitelioides, citoplasma anfofílico com núcleos ovalados. Tal achado é confirmado pelas características histológicas descritas pelo estudo de Subramanian et al. ${ }^{6}$

As mutações genéticas associadas, advêm dos genes KIT, os quais representam 95\% dos casos, e dos genes PDGFRa, que constituem $5 \%$ dos casos. Na macroscopia, a massa encontra-se na submucosa ou muscular própria, com crescimento expansivo para luz e a mucosa, pode estar intacta ou ulcerada com sangramentos. Já na microscopia, há presença de células fusiformes, citoplasma eosinofílico mal delimitado e núcleo ovoide. ${ }^{6}$

A imuno-histoquimica é o diagnóstico definitivo com a detecção da mutação no gene KIT (CD117+), receptor transmembrana presente em até $95 \%$ desses tumores mesenquimais. ${ }^{7}$ Tal fato, foi o que possibilitou o diagnóstico do caso, visto que o paciente apresentava positividade para este marcador.
O exame realizado ainda encontrou a presença de CD34+ que, aliado ao marcador já citado, fecha o diagnóstico. ${ }^{8}$

Ainda no mesmo exame, foi encontrada positividade para ki-67, sinalizador de atividade mitótica que, segundo o exame analisado, apresentava o equivalente a 2 mitoses por campo. $\mathrm{O}$ risco de agressividade do tumor é avaliado de acordo com o National Institutes of Health $(\mathrm{NIH})^{9}$, considerando os tamanho e atividade mitótica em: risco muito baixo (tamanho menor que $2 \mathrm{~cm}$ e menos de 5 mitoses), baixo risco $(2-5 \mathrm{~cm}$ e menos de 5 mitoses), risco intermediário (menor que 5 $\mathrm{cm}$ e 6-10 mitoses ou 5-10 cm e menos de 5 mitoses) e alto risco (maior que $5 \mathrm{~cm}$ e mais de 5 mitoses, maior de $10 \mathrm{~cm}$ e qualquer taxa de mitose ou qualquer tamanho e mais de 10 mitoses).

Tendo em vista que o tumor estudado apresentava o tamanho de $6,5 \mathrm{~cm}$ e taxa de 2 mitoses, pode-se dizer, que se classificava como sendo de risco intermediário. O tratamento desse tipo de tumor pode ser realizado com ressecção cirúrgica, podendo em alguns casos apresentar recidiva. Outro tipo de tratamento que a ser utilizado é a quimioterapia com mesilato de imatinibe e mesilato de sunitinibe. ${ }^{10}$

\section{CONCLUSÃO}

O tumor estromal gastrintestinal (GIST) deve ser reconhecido, pois são tumores mesenquimais mais comuns localizados no trato gastrointestinal. Torna-se necessário demonstrar a importância para a finalização do diagnóstico, visto que, a associação da história clínica dos pacientes, exames laboratoriais e de imagem, associados ao anatamopatológico ainda não fecham de forma definitiva o diagnóstico, necessitando de imunohistoquímico com pesquisa de CD-117 (c-kit).

Dessa forma, trata-se de uma doença já conhecida. No entanto, apresenta grande interesse ao cirurgião geral, pois, a constante 
atualização de achados permite conhecer as formas mais precisas de diagnóstico e oferecer ao paciente um tratamento moderno e mais eficaz.

\title{
HISTOPATHOLOGICAL CHARACTERISTICS OF A GASTROINTESTINAL STROMAL TUMOR
}

\section{ABSTRACT}

The gastrointestinal stromal tumor (GIST) is a type of tumor that can develop throughout the entire digestive tract. It has an unknown cause, its occurrence occurs in the different age groups, being more common in individuals of older age, the carrier of the disease is asymptomatic at the onset of the condition, developing symptoms only in more advanced stages. As it is considered a rather unusual tumor, it was considered the study of this pathology, in order to enlarge the knowledge about this morbidity, that is often unknown to health professionals. The aim of the study is to report histopathological findings of a patient diagnosed with Gastrointestinal Tumor of Stromal origin. Through data from the patient's medical record, and through the analysis of the histological slide, the histopathological findings were observed. The case report was carried out in a private pathology clinic in the city of João Pessoa - PB. The presence of elongated nucleus, tumor cells, in part of the lamina, was restricted to the muscular layer of the mucosa and, in part, invading the superjacent layer, besides counting, with necrosis area. The result could be confirmed by immunohistochemistry, which showed the presence of CD117 and CD34 markers. The association of the patient's clinical history, laboratory and imaging exams, together with the pathology did not definitively close the diagnosis, requiring immunohistochemistry with a CD-117 (c-kit) study. GIST is a silent tumor whose aggressiveness is variable and requires early diagnosis, while its study is limited, its detection will often continue to be late and will endanger patients' outcomes.
\end{abstract}

KEYWORDS: Oncology. Histology. Stroma.

\section{REFERÊNCIAS}

1 - Pantoja GKC, Saraiva KS, Castro Neto RG. Tumor estromal gastrointestinal do estômago concomitante a presença de hérnia inguinal com insinuação vesical: relato de caso. Braz. J. of Develop. Curitiba Fev, 2020; 6(2): 7546-59.

2- Vivi AAG, Antoniassi ACD, Coelho ACTER, Garetti B, Barbisan GG. Tumor estromal gastrointestinal de origem gástrica: relato de caso. Medicina (Ribeirao Preto. Online). São Paulo 2017; 50(3): 197-00.
3- Bejarano Glez-Serna D, Benjumea PG, Padilla ARR, García RB. Tumores del estroma gastrointestinal: revisión de nuestraexperiencia. Rev Chil de Cir. Ago. 2015; 67(4): 386-92.

4- Pacifico DSS, Cruz DAR, Souza LKM, Pinto ASB. Clinical and Diagnostic Presentation of Gastrointestinal Stromal Tumour (GIST): A 5-Year Follow-Up. REAS/EJCH, [periodic na internet] Ago 2019; 11(13): 1-8. 
5- De Macedo LL, Torres LR, Faucz RA, Tornin OS, Gonzalez FM, Aquino IM, et al. Tumor do estroma gastrintestinal: achados clínicos, radiológicos e anatomopatológicos. Radiol Bras. 2007; 40(3): 149-53.

6- Subramanian S, Sandhu AS, Balu J, Suresh P. Clinic-pathological aspect of gastro-intestinal stromal tumors at tertiary care Hospital India. J. of Coloproct. Mar 2020; 40(1): 12-19.

7- Alves GG; Penido BB; Sette CV; Rizzetto EA; dos Santos PE ; Manna EF et al. Tumor Estromal Extra-Gastrointestinal (E GIST): Relato de caso e revisão da literatura. Clin Onc Let. 2019; 2(2): 22-23.
8- Martins, MC; Longras C; Oliveria J; Santos A; Costa CS; Ferreira. Volumoso tumor do estroma gastrointestinal. Rev Portug de Cir. 2020; 46: 55-57.

9- Lim KT, \& Tan KY. Current research and treatment for gastrointestinal stromal tumors. World J of Gastroenterol, Jul 2017; 23(27): 4856-66.

10- Rodrigues JBSR, Campanati RG, Nolasco F, Bernardes AM, Sanches SRA, SavassiRocha PR. Redução tumoral pré-operatória do gist gástrico: a importância da terapia neoadjuvante. $A B C D$, Arq. Bras. Cir. Dig. Fev 2019; 32(1): e1427. 